GIANEZINI, M. et al. Notas sobre mudanças tecnológicas na produção e agroindústria de aves na região da Amrec, SC. PUBVET, Londrina, V. 8, N. 17, Ed. 266, Art. 1768, Setembro, 2014.

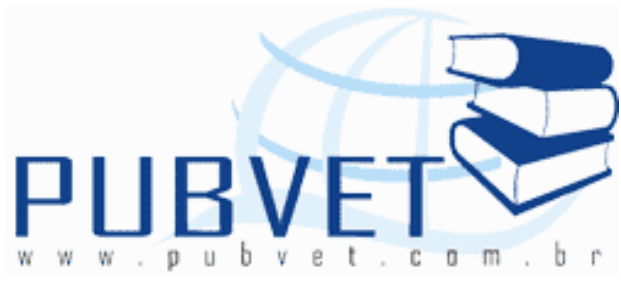

PUBVET, Publicações em Medicina Veterinária e Zootecnia.

\title{
Notas sobre mudanças tecnológicas na produção e agroindústria de aves na região da Amrec, SC
}

\author{
Miguelangelo Gianezini ${ }^{1}$, Lucas Nesi Destro ${ }^{2}$, Júlio César Zilli ${ }^{3}$, \\ Melissa Watanabe ${ }^{4}$
}

${ }^{1}$ Administrador. Doutor em Agronegócios. Professor do Programa de PósGraduação em Desenvolvimento Socioeconômico (UNESC).

${ }^{2}$ Graduando em Administração (UNESC), Bolsista PIBIC/CNPq/UNESC.

${ }^{3}$ Contador. Mestrando em Desenvolvimento Socioeconômico (UNESC).

${ }^{4}$ Eng. Agrônoma. Doutora em Agronegócios. Professora do Programa de Pós-Graduação em Desenvolvimento Socioeconômico (UNESC).

\section{Resumo}

O estudo de atividades agropecuárias e agroindustriais em regiões com economia diversificada é relevante pelo impacto desta produção no processo de desenvolvimento, contemplando realidades específicas e demonstrando como são produzidos seus principais bens agropecuários. Assim, objetivouse desenvolver uma pesquisa, voltada à temática da inovação (mudanças tecnológicas) nas atividades agropecuárias e agroindustriais, com foco no setor avícola dos municípios da AMREC, no Sul de Santa Catarina. O estudo teve abordagem qualitativa, sendo a variável temporal limitada ao segundo semestre de 2013 e a amostragem não probabilística intencional. Com relação aos procedimentos, optou-se: a) pela realização de pesquisa bibliográfica sobre a temática; b) pesquisa de campo, tanto para coleta de 
GIANEZINI, M. et al. Notas sobre mudanças tecnológicas na produção e agroindústria de aves na região da Amrec, SC. PUBVET, Londrina, V. 8, N. 17, Ed. 266, Art. 1768, Setembro, 2014.

informações junto aos expositores de uma feira agropecuária, quanto para coleta de dados com técnicos da CIDASC; e c) levantamento em documentos disponibilizado pela EPAGRI. Os resultados do estudo apontam que o crescimento da produção de aves na região tem atraído a atenção de algumas empresas. Contudo observou-se que as mesmas fabricam e ofertam equipamentos "de linha" e soluções tecnológicas ainda convencionais.

Palavras-chave: Tecnologia; Avicultura; Santa Catarina; AgroPonte.

\section{Notes on technological changes in the production and agribusiness poultry in the region of AMREC, Santa Catarina State, Brazil.}

\section{Abstract}

The study of agricultural and agro-industrial activities in areas with diversified economy is relevant because of the impact of this production in the local development. In this study we develop a research, on the theme of innovation (technological change) in agricultural and agro-industrial activities, focusing on the poultry production in the counties of AMREC region, southern of Santa Catarina State, Brazil. The research was conducted in 2013 and had a qualitative approach. We carried out: a) literature review on the subject, b) field research to collect information from the exhibitors in an agricultural fair (Agroponte) and with researcher from CIDASC, the agricultural development company of Santa Catarina State, and c) a survey in documents available from EPAGRI, the state agricultural research company. The study results indicate that the growth of poultry production in the region has attracted the attention of some companies. However, it was observed that these companies are offering the same machinery (product range) offered elsewhere and conventional technology solutions.

Keywords: Technology; Poultry; Santa Catarina; AgroPonte. 
GIANEZINI, M. et al. Notas sobre mudanças tecnológicas na produção e agroindústria de aves na região da Amrec, SC. PUBVET, Londrina, V. 8, N. 17, Ed. 266, Art. 1768, Setembro, 2014.

\section{INTRODUÇÃO}

O estudo de atividades agropecuárias e agroindustriais desenvolvidas em regiões com economia diversificada, como é o caso do Sul de Santa Catarina, implicam em maior complexidade, uma vez que a importância da produção de alimentos no processo de desenvolvimento econômico contemporâneo, precisa contemplar a inserção de novas tecnologias que permitam harmonizar a expansão (e sustentá-la ao longo do tempo) da atividade agropecuária e os aspectos que envolvem a preservação ambiental.

A região sul catarinense ocupa uma área de $9.608 \mathrm{~km}^{2}$, que compreende 43 municípios e uma população de aproximadamente $900 \mathrm{mil}$ habitantes, dentre os quais, cerca de 670 mil residentes em áreas urbanas. Ainda subdivide-se em três microrregiões, cada qual organizada por associação representativa: a Associação dos Municípios do Extremo Sul Catarinense (AMESC); a Associação dos Municípios da Região de Laguna (AMUREL) e a Associação dos Municípios da Região Carbonífera (AMREC), que reúne os municípios de Criciúma (maior pólo), Cocal do Sul, Forquilhinha, Içara, Lauro Muller, Morro da Fumaça, Nova Veneza, Orleans, Siderópolis, Treviso e Urussanga.

Do ponto de vista socioeconômico, o desenvolvimento da região esteve historicamente calcado na atividade carbonífera, aliado a atividades complementares que se desenvolveram em duas direções: Ao sul de Criciúma, com a exploração do carvão e a atividade agrícola; e ao norte de Criciúma, onde predominam atividades ligadas ao setor mineral, cerâmico, metal-mecânico, químico, agroindustrial e pesqueiro (SOUZA, 2012).

Assim, observa-se que no âmbito desta economia diversificada sempre houve espaço para as atividades agropecuárias e agroindustriais, que concorrem com outros setores pelo uso da terra, não sendo, contudo 
GIANEZINI, M. et al. Notas sobre mudanças tecnológicas na produção e agroindústria de aves na região da Amrec, SC. PUBVET, Londrina, V. 8, N. 17, Ed. 266, Art. 1768, Setembro, 2014.

predominantes, como em outras regiões, nem tão significativas quanto nos estados vizinhos do Paraná e do Rio Grande do Sul.

E neste contexto, o Sul de Santa Catarina - onde as interações entre a sociedade o ambiente levaram e levam a diferentes caminhos (históricos, econômicos e geográficos) no processo de mudança e uso da terra - tem se deparado com novos desafios e pressões por mudanças que permitam não apenas o aprimoramento das atividades agropecuárias, como também o atendimento a novos paradigmas socioeconômicos e ambientais.

Os municípios desta região foram escolhidos (com foco nos que compõem a AMREC), pois desde o início deste século têm buscado diversificar suas matrizes produtivas, integrando outras atividades a uma nova realidade do desenvolvimento socioeconômico do estado de Santa Catarina. Isto porque são reconhecidamente impactados pelas obras de duplicação do Eixo-Sul da BR-101, tanto que, a cada ano, é possível observar o resultado deste processo dinâmico transformando o panorama do Sul Catarinense, considerado até pouco tempo, uma região de exclusiva exploração mineral.

Por isso, espera-se que este estudo, ainda que preliminar, possa contribuir tanto para a área de investigação quanto para o setor produtivo local, uma vez que é integrante dos resultados do projeto de pesquisa "Desenvolvimento e inovação na agropecuária e agroindústria do sul catarinense: Mudanças tecnológicas nos municípios integrantes da AMREC".

O estudo teve por objetivo compreender e apresentar as principais mudanças tecnológicas das atividades agropecuárias e agroindustriais, com foco na avicultura. O intuito foi o de analisar mudanças recentes e fornecer subsídios para o aprimoramento da gestão das organizações (públicas ou privadas) que participam desta cadeia produtiva nos municípios que compõe a AMREC, no sul de Santa Catarina. 
GIANEZINI, M. et al. Notas sobre mudanças tecnológicas na produção e agroindústria de aves na região da Amrec, SC. PUBVET, Londrina, V. 8, N. 17, Ed. 266, Art. 1768, Setembro, 2014.

\section{METODOLOGIA}

Este estudo teve abordagem predominantemente qualitativa, por se tratar de artigo integrante de projeto de pesquisa com ênfase na compressão da condição das atividades agropecuárias e agroindustriais, pautadas pela observação e descrição deste processo nos município da região da AMREC.

A variável temporal foi limitada aos seis meses do segundo semestre do ano de 2013, considerando o cronograma da proposta de pesquisa geral (PIBIC/UNESC); e a amostragem foi não probabilística intencional, devido ao caráter dos expositores da feira agropecuária visitada e ao grupo definido de municípios que compõe a AMREC.

Com relação aos procedimentos, optou-se: a) pela realização de pesquisa bibliográfica preliminar sobre a temática, que foi integrada aos resultados do estudo); b) por pesquisa de campo, tanto para coleta de informações junto aos expositores de uma feira agropecuária (AgroPonte, em 2013), quanto para coleta de dados com técnicos da Companhia Integrada de Desenvolvimento Agrícola de Santa Catarina (CIDASC); e c) por levantamento em documentos disponibilizado pela Empresa de Pesquisa Agropecuária e Extensão Rural de Santa Catarina (EPAGRI).

\section{RESULTADOS E DISCUSSÃO}

Destacam-se a seguir considerações sobre a inovação e mudança tecnológica, produção animal e finalmente um panorama da produção de aves e tecnologia aplica na AMREC.

\subsection{Considerações sobre inovação e mudança tecnológica}

A inovação refere-se, de maneira geral, a uma ideia, método ou objeto que é criado e difere-se dos padrões anteriores. Uma das definições 
GIANEZINI, M. et al. Notas sobre mudanças tecnológicas na produção e agroindústria de aves na região da Amrec, SC. PUBVET, Londrina, V. 8, N. 17, Ed. 266, Art. 1768, Setembro, 2014.

de inovação mais aceitas caracteriza-a como a busca, descoberta, experimentação, desenvolvimento, imitação e adoção de novos produtos, processos e novas técnicas organizacionais (DOSI, 1988). Os agentes envolvidos no processo novo devem possuir algum tipo de percepção quanto às oportunidades técnicas e econômicas de algo que ainda não foi explorado.

$\mathrm{Na}$ percepção da administração e das organizações, a inovação é entendida como instrumento específico dos empreendedores, o meio pelo qual eles exploram a mudança como uma oportunidade para um negócio ou serviço diferente (DRUCKER, 2001).

Por conseguinte, dentre as "possibilidades de inovação", aquelas que relacionadas ao produto ou processo são entendidas como "inovações tecnológicas". De acordo com um dos precursores da área (SCHUMPETER, 1982), isto significa que um produto "tecnologicamente novo" é um produto cujas características tecnológicas ou usos pretendidos "diferem significativamente dos produtos previamente produzidos, pois deriva do uso de novos conhecimentos". "Um produto tecnologicamente melhorado é um produto existente, cujo desempenho foi significativamente aperfeiçoado" (SCHUMPETER, 1982).

Já a inovação tecnológica de processo, mais contemporânea, é entendida como a adoção de métodos de produção tecnologicamente novos ou significativamente melhorada, incluindo métodos de apresentação de produto. Esses métodos podem envolver mudanças em equipamentos, na organização da produção, ou a combinação dessas mudanças, e podem ser derivados do uso de novo conhecimento (OCDE/EUROSTAT, 1997).

$\mathrm{Na}$ seara da produção agropecuária e agroindustrial, o tema da inovação ainda não foi suficientemente explorado e as ações dos stakeholders têm sido pautadas por preocupações de ordem econômica, como competitividade, pressões de mercado e investimento, além da recente - porém ainda não determinante - demanda de produção de alimentos com menor impacto ambiental. 
GIANEZINI, M. et al. Notas sobre mudanças tecnológicas na produção e agroindústria de aves na região da Amrec, SC. PUBVET, Londrina, V. 8, N. 17, Ed. 266, Art. 1768, Setembro, 2014.

\subsection{Mudança tecnológica e produção animal}

As considerações teóricas sobre inovação e produção de novas tecnologias, quando aplicadas, revelam disparidades em algumas áreas como a da produção animal. Isto porque os diferentes tipos de sistema produtivos possuem ritmos próprios, que modificam-se de acordo com demanda de cada setor. Por conseguinte, pode-se mencionar que a mudança tecnológica na produção animal passa pelo aprimoramento da tecnologia de insumos (produto) e tecnologia de processos (gestão do conhecimento).

Foi na década 1960 que surgiu em Santa Catarina o sistema integrado, modelo adotado atualmente em todo o país. Anteriormente, a avicultura era feita por granjeiros que eram responsáveis por toda a cadeia produtiva. No período de 1990 com a abertura econômica surge um formato estratégico de produção que busca redução de custos, tecnologia e um aumento na industrialização dos produtos (DAMBRÓS JR., 2010).

Com relações aos inputs desta atividade, um exemplo de tecnologia de insumos pode ser encontrado na descrição de Barcellos et al (2011, p. 193):

O crescimento em produtividade é resultado do uso de poucos insumos, em geral resultantes dos avanços científicos; novas linhagens, aditivos nutricionais, vacinas, ingredientes, promotores do crescimento, instalações e equipamentos - são capazes de mudar rápida e economicamente os níveis de produtividade. Todos eles são comercializáveis, introduzidas com facilidade na produção, desenvolvidos e gerenciados por poucos agentes são as tecnologias de insumos (produtos).

Tais tecnologias são disponibilizadas a muitos avicultores, com reflexos sistêmicos na produtividade de uma determinada localidade, como a região estudada. Isto explica o crescimento da avicultura brasileira em relação aos seus competidores internos (suinocultura, bovinocultura) e externos (demais países produtores de proteína animal). Adicionalmente a isto, tem-se a tecnologia de processos, menos impactante que a de insumos, 
GIANEZINI, M. et al. Notas sobre mudanças tecnológicas na produção e agroindústria de aves na região da Amrec, SC. PUBVET, Londrina, V. 8, N. 17, Ed. 266, Art. 1768, Setembro, 2014.

que envolve o manejo do aviário, da alimentação, da sanidade, da ambiência, entre outros.

Barcellos et al (2011) ainda comentam que poucas as empresas do setor realizam pesquisas, geram insumos e os colocam à venda. A concorrência as torna mais competitivas e eficientes na disputa do cliente e na valorização da inovação. Com isto os avanços são rápidos e focados exclusivamente no resultado econômico imediato.

Exemplos significativos deste processo de análise do cliente final é a produção com o foco nos mercados específicos, como por exemplo, para o mercado nacional a diminuição do tamanho do (frango inteiro para famílias menores), mercado externo (frangos inteiros maiores), bem como especificidades religiosas sendo respeitadas (método de abate halal) na busca de mercados consumidores com tais exigências

\subsection{Panorama do objeto de estudo na AMREC}

Com o intuito de apresentar um panorama da mudança tecnológica e produção de aves na AMREC, optou-se, como ponto de partida, por um levantamento junto aos expositores da AgroPonte 2013.

Trata-se de uma feira anual, realizada em Criciúma-SC, que reúne empresas do agronegócio e produtores familiares, visando agrupar, integrar, relacionar e promover o desenvolvimento individual e coletivo dos setores da avicultura, agricultura, bovinocultura, suinocultura, apicultura, fruticultura, piscicultura, turismo de negócios e outros por meio de ações para orientação tecnológica, preparos, gerenciamento, produção, logística, transformação, industrialização e comercialização (EMTURSUL, 2012).

A terceira edição da AgroPonte foi realizada de 14 a 18 de agosto de 2013 com exposição de máquinas, equipamentos, insumos e tecnologias para produção agropecuária, além de exposição de animais, com participação de mais 150 espécies diferentes de aves, bovinos, equinos, suínos, caprinos, caninos, ovinos, felinos, peixes e pássaros. O evento 
GIANEZINI, M. et al. Notas sobre mudanças tecnológicas na produção e agroindústria de aves na região da Amrec, SC. PUBVET, Londrina, V. 8, N. 17, Ed. 266, Art. 1768, Setembro, 2014.

fomenta os produtos produzidos nas propriedades familiares, que formam mais de 30 cooperativas, além da vitrine para pequenas, medias e grandes empresas apresentarem seus produtos e equipamentos (EMTURSUL, 2013).

Assim, tendo em vista o foco do estudo, após observação e apresentação da feira, buscou-se sintetizar no Quadro 1 os resultados do levantamento realizado junto as empresas ligadas a avicultura.

Quadro 1 - Setores de tecnologia para avicultura presentes na AgroPonte.

\begin{tabular}{|l|c|c|}
\hline \multicolumn{1}{|c|}{ SETOR } & SEDE & NOVIDADES NA FEIRA \\
\hline $\begin{array}{l}\text { Equipamentos Aves/suínos/gado leiteiro, } \\
\text { armazenagem }\end{array}$ & Marau-RS & Produtos de linha \\
\hline Setor Avícola/Climatização & Israel & Produtos de Linha \\
\hline Refrigeração & Tubarão-SC & Produtos de linha \\
\hline Refrigeração, alimentação/automação Avícola & São Paulo-SP & Produtos de linha \\
\hline Equipamentos Aves/suínos & Nova Veneza-SC & \\
\hline Aquecedores, Equipamentos agrícolas & Orleans-SC & Produtos de linha \\
\hline Equipamentos Aves/Suínos & Matelândia-PR & Tratamento de Resíduos \\
\hline
\end{tabular}

Fonte: Elaboração Própria.

De acordo com o Quadro 1 percebe-se que a AgroPonte conta com a presença de setores diversificados, no qual as atividades agropecuárias possuem destaque. Além da presença de empresas pertencentes aos municípios do Sul de Santa Catarina, como Tubarão, Nova Veneza e Orleans, os estados do Paraná, Rio Grande do Sul e São Paulo também registram a sua participação. Vale ressaltar também a presença de uma empresa com sede internacional (Israel), pertencente ao setor avícola/climatização.

Contudo, observa-se que os produtos expostos na feira são aqueles considerados como "de linha", sendo que as máquinas, equipamentos e insumos são predominantemente voltados a avicultura, juntamente com o 
GIANEZINI, M. et al. Notas sobre mudanças tecnológicas na produção e agroindústria de aves na região da Amrec, SC. PUBVET, Londrina, V. 8, N. 17, Ed. 266, Art. 1768, Setembro, 2014.

cultivo de arroz, cujas análises das inovações na cadeia produtiva na região foram estudas por Vieira et at (2012).

Por conseguinte, este panorama pode ser constatado na observação dos estabelecimentos de produção e comercialização de aves presentes na AMREC, como destacado no Quadro 2.

Quadro 2 - Estabelecimentos de produção e comercialização de aves.

\begin{tabular}{|l|c|c|}
\hline \multicolumn{1}{|c|}{ ESTABELECIMENTO } & SETOR & MUNCÍPIO \\
\hline Proave Agroindustrial & Produção e criação & Içara \\
\hline Mercoaves & Produção e criação & Urussanga \\
\hline Lema & Produção e criação & Urussanga \\
\hline Galla & Produção e criação & Orleans \\
\hline Avícola Catarinense & Produção e criação & Lauro Muller \\
\hline JBS unidade Nova Veneza & Frigorífico/Abate & Nova Veneza \\
\hline Seara Alimentos & Frigorífico/Abate & Forquilhinha \\
\hline PASOVOS Pasteurizadora de ovos & Outros & Forquilhinha \\
\hline
\end{tabular}

Fonte: Elaboração Própria.

A produção e criação de aves é destaque na AMREC, uma vez que dentre os oito estabelecimentos, cinco correspondem a este tipo de operação e duas unidades (JBS e Seara) operam no abate e comercialização, conforme mencionado no Quadro 2. Percebe-se também que dentre os 11 municípios que fazem parte da AMREC, seis municípios, dentre estes Içara, Urussanga, Orleans e Lauro Muller estão focados na produção e criação e Nova Veneza e Forquilhinha com frigoríficos e abate de aves.

A presença destes estabelecimentos, por sua vez, indica uma interdependência, em especial quando observados os dados sobre a produção de aves na região da AMREC, como apresentado na Tabela 1. 
GIANEZINI, M. et al. Notas sobre mudanças tecnológicas na produção e agroindústria de aves na região da Amrec, SC. PUBVET, Londrina, V. 8, N. 17, Ed. 266, Art. 1768, Setembro, 2014.

Tabela 1 - Propriedades de produção de aves na AMREC.

\begin{tabular}{|c|c|c|c|}
\hline MUNICÍPIO & $\begin{array}{c}\text { Propriedades } \\
\text { com granjas de } \\
\text { aves }\end{array}$ & FINALIDADE & $\begin{array}{l}\text { NÚMERO DE } \\
\text { ANIMAIS }\end{array}$ \\
\hline Cocal do Sul & 7 & Corte & 109.069 \\
\hline Cocal do Sul & 2 & Matriz de Corte & 39.904 \\
\hline Criciúma & 12 & Corte & 5.276 \\
\hline Criciúma & 1 & Matriz de corte & 13.608 \\
\hline Forquilhinha & 34 & Corte & 297.506 \\
\hline Içara & 17 & Corte & 216.177 \\
\hline Içara & 9 & Matriz de Corte & 162.616 \\
\hline Lauro Muller & 36 & Corte & 743.868 \\
\hline Lauro Muller & 11 & Matriz de Corte & 106.474 \\
\hline Morro da Fumaça & 6 & Corte & 58.040 \\
\hline Morro da Fumaça & 1 & Matriz de Corte & 15.368 \\
\hline Nova Veneza & 64 & Corte & 565.914 \\
\hline Nova Veneza & 6 & Matriz de Corte & 13.990 \\
\hline Orleans & 79 & Corte & 780.039 \\
\hline Orleans & 51 & Matriz de Corte & 510.087 \\
\hline Siderópolis & 73 & Corte & 432.934 \\
\hline Siderópolis & 10 & Matriz de Corte & 49.960 \\
\hline Treviso & 36 & Corte & 420.415 \\
\hline Treviso & 5 & Matriz de Corte & 14.880 \\
\hline Urussanga & 37 & Corte & 455.712 \\
\hline Urussanga & 6 & Matriz de Corte & 35.434 \\
\hline
\end{tabular}

Fonte: Elaborado a partir de dados fornecidos pela CIDASC, setembro de 2013. 
GIANEZINI, M. et al. Notas sobre mudanças tecnológicas na produção e agroindústria de aves na região da Amrec, SC. PUBVET, Londrina, V. 8, N. 17, Ed. 266, Art. 1768, Setembro, 2014.

De acordo com a Tabela 1, observa-se que a produção de aves de corte totaliza 401 propriedades com a 4.084 .950 animais. Com relação à matriz de corte, verifica-se uma totalidade de 102 propriedades com 962.321 animais. Em ambas as finalidades, Orleans destaca-se como sendo o município que possui o maior número de propriedades com granjas e também número de animais.

Estes números atuais compõem os resultados do crescimento da produção de aves na AMREC em 2010, que representava 3,1\% do total de aves em Santa Catarina e cresceu para 4,7\% em 2012 de acordo com dados da síntese anual da agricultura de Santa Catarina (CEPA/EPAGRI, 2012).

Os resultados observados também contribuem para ratificar o Sul de Santa Catarina como o principal mercado consumidor final da produção industrial e familiar da região, sendo que, a indústria frigorífica para a carne de frangos, que abastece o País e importantes mercados asiáticos, europeus, africanos e sul americanos (EMTURSUL, 2012).

\section{CONSIDERAÇÕES FINAIS}

O tempo dinâmico de produção - mais curto, quando comparado com outras atividades pecuárias - permite que a criação e aves avance com maior rapidez em diversos ambientes. No caso deste estudo, observou-se que este fenômeno tem ocorrido na região da AMREC, onde há perspectiva de crescimento e desenvolvimento do setor. Observou-se que a presença de grandes empresas do ramo (em especial as de abate) contribui para este quadro.

Os principais resultados obtidos na Agroponte e junto a CIDASC e EPAGRI (organizados em quadros e tabela), indicam que o crescimento da produção de aves na região também tem atraído a atenção de empresas fabricantes de equipamentos e insumos. Contudo constatou-se que as mesmas fabricam e ofertam equipamentos "de linha" (catálogo) e soluções 
GIANEZINI, M. et al. Notas sobre mudanças tecnológicas na produção e agroindústria de aves na região da Amrec, SC. PUBVET, Londrina, V. 8, N. 17, Ed. 266, Art. 1768, Setembro, 2014.

tecnológicas consideradas convencionais. Desta forma as novidades (mudanças tecnológicas) disponíveis para os produtores locais, dificilmente são utilizadas de forma sistemática, pois exigem, de acordo com o referencial estudado, quebras de paradigmas e mudanças comportamentais, as vezes radicais, devido à complexidade e diversidade de suas implicações.

Por fim, acredita-se que o estudo, dentro de suas limitações, tenha contribuído enquanto proponente de novo objeto de pesquisa do desenvolvimento agropecuário e agroindustrial na região da Amrec, complementando os estudos teóricos e aplicados já realizados e ou em andamento.

\section{Agradecimentos:}

Fontes Financiadoras: Programa Institucional de Bolsas de Iniciação Científica CNPq/UNESC e Grupo de Pesquisa Propriedade Intelectual, Desenvolvimento e Inovação (PIDI).

\section{REFERÊNCIAS}

BARCELLOS, J. O. J. et al. Technological innovation and entrepreneurship in animal production. Revista Brasileira de Zootecnia, Viçosa, v. 40, p. 189-200, 2011.

DAMBRÓS JÚNIOR, D. Produção de ovos no Brasil. Central de Inteligência de Aves e Suínos. Concórdia, SC. Disponível em: <http://www.cnpsa. embrapa.br/cias/index.php?option=com_content\&view $=$ article\&id=15\&Itemid $=17>$. Acesso em: 09/11/2013.

DOSI, G. The nature of the innovative process. In: DOSI, G.; FREEMAN, C.; NELSON, R. (Eds.). Technical change and economic theory. 1. ed. London: Pinter Publishers, 1988. p. 221-238.

DRUCKER, P. O melhor de Peter Drucker. Nobel, 2001.

EMTURSUL - Convention \& Visitors Bureau, Criciúma e Região. Documento Eletrônico, 2012. Disponível em: <http://eventos.santur.sc.gov.br/index. php?view=details\&id=888:agroponte-agronegocios-e-agricultura-familiar $\quad$ \&pop=1\& tmpl=component>. Acesso em: 15 nov. 2013.

EMTURSUL - Convention \& Visitors Bureau, Criciúma e Região. Documento Eletrônico, 2013. Disponível em: <http://eventos.santur.sc.gov.br/index. php? view $=$ details\&id=1345:3o-agroponte-agronegocios-e-agricultura-familiar $>$. Acesso em: 20 dez. 2013. 
ORGANISATION FOR ECONOMIC CO-OPERATION AND DEVELOPMENT - OCDE/ EUROSTAT. Manual de Oslo - Diretrizes para coleta e interpretação de dados sobre inovação. 1997. Disponível em: <http://www.finep.gov.br/ imprensa/sala_imprensa/manual_de_oslo.pdf>. Acesso em: 12 jul 2013.

SCHUMPETER, J. A. Teoria do desenvolvimento econômico: Uma investigação sobre lucros, capital, crédito, juro e o ciclo econômico. São Paulo: Abril cultural, 1982.

SOUZA, G. C. de. A emergência de novas áreas do conhecimento científico para a problemática socioambiental: o caso da engenharia ambiental e sua contribuição no contexto da região carbonífera catarinense. 2012197 f. Dissertação (mestrado). PPGCA/UNESC. Criciúma, 2012.

VIEIRA, A. C. P. et al. Análise das Inovações na Cadeia Produtiva do Arroz na Região Sul Catarinense: AMESC e AMREC. In: III Seminário das Ciências Sociais Aplicadas, 2012, Criciúma. III Seminário das Ciências Sociais Aplicadas. Criciúma: UNESC, 2012.

CENTRO DE SOCIOECONOMIA E PLANEJAMENTO AGRÍCOLA - CEPA, EPAGRI. Síntese anual da agricultura de Santa Catarina 2009-2010. Disponível em:

<http://cepa.epagri.sc.gov.br/Publicacoes/Sintese_2010/sintese\%2020 10_inteira.pdf>. Acesso em: 08 out. 2013. 